\title{
El psicólogo educativo y su formación académica desde la perspectiva del desarrollo
}

Alba Yanalte Álvarez Mejía, ${ }^{1}$ Germán Pérez Estrada ${ }^{1}$ y Rosa María Nashiki Angulo ${ }^{2}$

\section{Introducción}

El presente documento pretende caracterizar el tema del desarrollo como lo organizaron los académicos en la línea curricular Desarrollo y Aprendizaje (LCDA) en el plan 2009. Para ello, se describen los contenidos más relevantes de las asignaturas que conforman esta línea y su contribución en la formación de psicólogos educativos a partir de las perspectivas del desarrollo y los procesos de cambio desde la infancia hasta la vejez, así como la influencia del ambiente en estos procesos.

Dichos elementos son básicos para la comprensión de las personas como parte de grupos sociales que cuentan con individualidades que requieren ser comprendidas para resaltar los procesos $\mathrm{y}$, en consecuencia, partir de esto para implementar intervenciones $\mathrm{o}$ acciones acordes a dichas individualidades.

2 Universidad Nacional Autónoma de México, Facultad de Psicología. 


\section{El desarrollo}

El desarrollo se entiende como un proceso continuo (Pastor, Nashiki y Pérez, 2013), con las siguientes características:

- Universal, porque se presenta de la misma forma en todas las personas independientemente de su nacionalidad, condición social, creencias, cultura y tiempo.

- Secuencial, ya que las habilidades se van dominando en el mismo orden.

- Jerárquico, es decir, las habilidades iniciales y más básicas son el cimiento para la adquisición de las más complejas.

- Dinámico, porque nunca se estanca y está en constante movimiento.

No obstante lo anterior, existen factores que influyen en la manera en que se presenta el proceso de desarrollo en una persona, por ejemplo:

- El ambiente en que se desenvuelve, éste puede presentar elementos que promuevan o limiten la adquisición de habilidades.

- La biología, que determina las posibilidades y ritmo de desarrollo.

- La interacción con las personas cercanas, ya que las relaciones interpersonales pueden promover o limitar el desarrollo.

- Las habilidades desarrolladas, como base para adquirir nuevas habilidades que contribuyen a la preparación de la persona para poner en práctica y dominar nuevas habilidades.

De esta manera, se plantea que el desarrollo se interrelaciona con el aprendizaje y ninguno se puede dar sin el otro, además de que cualquier avance que se presente en el primero afectará el segundo (Pastor, Nashiki y Pérez, 2013). Es decir, los procesos de desarrollo son dinámicos y, si se consideran las 
características anteriores, se pueden brindar alternativas de intervención acordes a características personales.

\section{Línea curricular. Desarrollo y aprendizaje}

Bajo esta idea, la decisión respecto a qué contenidos incluir en las materias que abordan el tema del desarrollo en esta LC, se basó en los resultados del estudio de egresados titulados con relación a los contenidos relevantes para desempeñar actividades profesionales, formación recibida y lugar donde laboran (Hernández, Rubio y Villaseñor, 2009), así como en las características del perfil de ingreso de los estudiantes a este programa educativo (Lozano y Rodríguez, 2005).

De acuerdo con Bernstein (1988), el rediseño, programación y desarrollo de un plan de estudios depende del trabajo y la colaboración entre los docentes involucrados en dicha tarea, de tal manera que las intenciones, organización, trabajo curricular y uso de estrategias permita a los estudiantes construir sus conocimientos vinculando los contenidos de las materias y los propósitos de las Lc desde una visión amplia.

También se consideró la reflexión de Coll (1990) respecto a concebir la Psicología Educativa como una disciplina aplicada en la que los conocimientos básicos pueden usarse para explicar y predecir, en tanto los conocimientos prácticos podrían enfocarse en intervenir, evaluar y colaborar.

En este sentido, el grupo de docentes que participa en esta Lc, argumentó la importancia de que el psicólogo educativo reconozca el desarrollo humano en todo el ciclo vital como un continuo vinculado al contexto en que interactúan los sujetos con materiales, personas y situaciones que guían su desarrollo, su aprendizaje y donde el mismo sujeto también genera cambios. Estos cambios no terminan en la adolescencia, sino que continúan hasta la vejez y se organizan en diferentes áreas para su mejor estudio y comprensión.

Para entender esta visión, se incorporaron en el programa de la asignatura Psicología Evolutiva de la Infancia, los modelos 
explicativos organicista, mecanicista y sociocultural, esto permite comprender el desarrollo desde el aspecto individual y en su relación con lo social. Las áreas del desarrollo que se revisan son: a) cognitivo, enfatizando periodos y mecanismos de aprendizaje, así como el juego y sus reglas; b) afectivo, analiza aspectos sobre apego y conocimiento de sí mismo, y c) lenguaje, centrándose en sus componentes, su adquisición y sus etapas de desarrollo. La formación de los estudiantes al revisar esta etapa evolutiva se centra en lograr una clara relación entre desarrollo e interacción de los sujetos en sus diversos entornos.

En la asignatura Psicología Evolutiva de la Adolescencia, se conceptualiza esta última a partir de una revisión histórica y teórica como un "invento sociocultural" actual y los cambios que ésta implica en las áreas: a) anatómica y fisiológica, enfatizando en las consecuencias psicológicas de la pubertad temprana y tardía; b) cognitiva, al revisar sus características, funciones y estructura formal del pensamiento; c) moral, centrándose en las normas, "lo bueno y lo malo", los estadios del razonamiento moral y normas prosociales, y d) psicosocial desde dos posturas: la primera, a partir de los grupos sociales de referencia, las manifestaciones culturales, expectativas y relación entre iguales; y la segunda, enfatizando los problemas que pueden surgir en la interacción en estos grupos, por ejemplo: embarazo, prácticas en torno a la sexualidad, trastornos alimentarios y abuso de sustancias.

El curso Psicología Evolutiva de la Adultez y Vejez enfatiza el sentido continuo del ciclo vital, se revisan distintas concepciones sobre adultez y vejez, sus subetapas (temprana, media y tardía) atendiendo a las diferencias históricas y culturales. Las áreas que se trabajan son: a) salud y sexualidad, para analizar cómo afectan y su relación con estilos de vida llevados desde la infancia; b) social y afectiva, enfatiza el significado de los distintos roles que se asumen (matrimonio, paternidad, abuelidad, divorcio, nido vacío y muerte), los cambios en el contexto familiar, laboral y profesional (empleo, despido, jubilación), las relaciones sociales (amistades) y el proceso de duelo; c) cognitiva y aprendizaje, centrada en los cambios que ocurren en los sistemas sensoriales y perceptuales, memoria, atención y pensamiento posformal para 
entender las condiciones en que se lleva a cabo el proceso de aprendizaje en estas etapas y realizar propuestas acordes, ya que los cambios no implican solamente deterioro o pérdidas, sino también pueden desplegarse ciertas funciones psicológicas y vitales.

Bajo esta idea, se pretende que los psicólogos educativos aprendan a buscar explicaciones tanto para el desarrollo como para el aprendizaje desde diferentes posturas teóricas o modelos que permiten una mejor comprensión de los sujetos, al entender sus necesidades, problemas, formas de aprender y de desarrollarse, los cuales cambian conforme pasan los años para dar cabida a explicaciones diversas y complejas del mismo suceso.

\section{La participación docente en la formación del psicólogo educativo}

En el proceso de construcción de los cursos del plan 2009, los docentes consideraron importante formar a los estudiantes bajo la idea de construir sus saberes en grupo con la guía de un mediador, ya que es insuficiente tener un cúmulo de información; se requiere también la habilidad de usarla, ajustándola a los contextos, necesidades y población atendida.

Esto ha generado que la organización de los contenidos y la proyección de los mismos sea modificada hacia la reflexión y análisis de cada etapa evolutiva, lo cual lleva al profesional en formación a comprender los procesos de cambio de cada una de ellas, así como las posibles necesidades de intervención educativa como parte de los retos del desarrollo y del momento sociohistórico en el que se encuentra el sujeto.

De ahí que se plantea la formación del psicólogo educativo desde la consideración de los contextos educativos y su relación con las prácticas educativas propias de éstos; es decir, se busca que el estudiante centre su atención en los procesos de desarrollo desde una perspectiva contextual y social, y no solamente en la atención individual y escolarizada.

En este contexto, el papel del psicólogo educativo no se reduce a juzgar o criticar, sino a comprender una situación 
con base en los avances de la ciencia y a identificar aquellos elementos que favorecen el desarrollo y el aprendizaje de sus miembros desde la escuela, la familia y la comunidad. Es así como aprende que sus propuestas requieren ser contextualizadas para lograr mejor aceptación y resultados.

Estas ideas han generado un cúmulo significativo de información para explicar el desarrollo en los diferentes momentos de la vida del ser humano y entender la diversidad de grupos que existen, los cuales comparten aspectos generales, pero tienen características propias que los hacen diferentes y particulares del resto. Estas visiones implican la formación de psicólogos educativos sensibles a los procesos, capaces de llevar a cabo intervenciones que respondan a las particularidades de los individuos y sus contextos, desde una visión más centrada en los procesos que en los productos.

Para lograr lo anterior, se proponen actividades prácticas a lo largo de los tres cursos y se analizan a partir de la información revisada, enfatizando en aspectos propios de los participantes, el momento histórico y elementos socioculturales de sus grupos de referencia. Esto permite generar en los estudiantes una visión más completa del desarrollo humano.

\section{Conclusiones}

Resulta interesante, en la formación de los psicólogos educativos, la revisión sobre la transformación del concepto desarrollo humano a lo largo de la vida y cómo estos cambios se construyen rodeados de factores sociales, de salud, educativos, culturales, que exige la flexibilidad de pensamiento y el trabajo interdisciplinario para hacer propuestas de atención, prevención o mejora adecuadas a los procesos mismos de cada etapa del ciclo vital, al tiempo que contribuye en la conceptualización del individuo como sujeto que modifica el contexto sociocultural que le influye.

Esto abre posibilidades laborales para el psicólogo educativo más allá del aula, ya que si bien es importante identificar 
y reconocer situaciones propias de cada sujeto, entenderlas de manera aislada limita su actuar, en tanto incorporar variables para explicarlo le permite un margen mayor de actuación.

Es así como esta Lc contribuye al logro del perfil de egreso, al abordar los conocimientos psicológicos sobre los procesos de desarrollo humano, especialmente aquellos derivados de su participación en los distintos niveles educativos y los relacionados con las prácticas socioculturales, de tal manera que el psicólogo educativo coadyuve en la construcción de programas de apoyo psicopedagógico dirigidos a distintos niveles educativos para mejorar los aprendizajes y favorecer diversas áreas del desarrollo, así como también en los conocimientos y las habilidades para colaborar con otros profesionales de la educación, participar con actitudes propositivas y de crítica constructiva, de respeto a los distintos actores educativos y de servicio para mejorar de manera reflexiva los procesos de enseñanza y de aprendizaje en las comunidades educativas.

\section{Referencias}

Bernstein, B. (1988). Clase, códigos y control. Madrid: Morata. Coll, C. (1990). Concepciones y tendencias actuales en psicología de la educación. En C. Coll, J. Palacios y A. Marchesi (Comps.). Desarrollo psicológico y educación. Psicología de la educación escolar, 29-64. Madrid: Alianza.

Furlán, A., y Remedi, E. (1979). Aportaciones a la didáctica de la educación superior. Construcción de la estructura metodológica de base. México: ENEP-IZTACALA-UNAM, Departamento de Pedagogía.

Hernández, J., Pérez, C., Martínez, G., Bollás, P. y Dzib, A. (2009). Propuesta del Plan de Estudios de la Licenciatura en Psicología Educativa. Plan 2009. Universidad Pedagógica Nacional.

Hernández, J., Rubio, M. y Villaseñor, S. (2009). Estudio sobre las competencias de los egresados en Psicología Educativa de la Universidad Pedagógica Nacional Unidad Ajusco: 
Avances de resultados. Mención del X Congreso de Investigación Educativa. México: comie.

Lozano, M. y Rodríguez, M. (2005). Perfil de ingreso: serie histórica 1995-2003. Estudios sobre la UPN. México: UPN.

Pastor, R., Nashiki, R. y Pérez, M. (2013). El desarrollo y aprendizaje infantil y su observación. Compendio de lecturas de información básica para educadoras. México: Puentes para Crecer, Facultad de Psicología, UNAM. 